\title{
Periocular rejuvenation with neurotoxin and dermal filler
}

\author{
Wendy W. Lee, Alexandra E. Levitt \\ Bascom Palmer Eye Institute, University of Miami, Miami, FL 33136, USA.
}

Correspondence to: Wendy W. Lee, Bascom Palmer Eye Institute, University of Miami, 900 NW 17th Street, Miami, FL 33136, USA. E-mail:wlee@med.miami.edu

How to cite this article: Lee WW, Levitt AE. Periocular rejuvenation with neurotoxin and dermal filler. Plast Aesthet Res 2018;5:43. http://dx.doi.org/10.20517/2347-9264.2018.39

Received: 26 May 2018 First Decision: 28 Jul 2018 Revised: 11 Oct 2018 Accepted: 16 Oct 2018 Published: 23 Oct 2018

Science Editor: Raúl González-García Copy Editor: Cai-Hong Wang Production Editor: Zhong-Yu Guo

\begin{abstract}
The periocular area is one of the first regions of the body to show signs of aging, which include static and dynamic rhytids as well as subcutaneous volume loss. The complex anatomy and dynamic underlying muscles of facial expression make this region particularly difficult to treat. Botulinum toxins and fillers, especially when used in combination, offer an excellent approach to minimally invasive rejuvenation of this area. This article aims to present a basic overview and clinical primer for the use of these injectables along with advice on avoiding and managing common complications.
\end{abstract}

Keywords: Botox, botulinum toxin, fillers, hyaluronic acid, rejuvenation, periorbital, aesthetics

\section{FEATURES OF AND FACTORS AFFECTING PERIOCULAR AGING}

Typical features of facial aging include changes in skin pigmentation, increased skin laxity, rhytid formation, and volume loss ${ }^{[1,2]}$. The periorbital region is particularly vulnerable to the effects of aging based on intrinsic properties of these tissues. For one, eyelid skin is among the thinnest in the body, at around $0.3-0.5 \mathrm{~mm}^{[3]}$. It lacks underlying subcutaneous fat to mitigate the contour defects and fat prolapse attendant with age. The delicate nature of this tissue also readily transmits underlying pigments, including blood products, muscle, and vessels. There is less collagen and elastin than surrounding skin, rendering periorbital skin less resilient and prone to early rhytid formation. In addition, eyelid and periocular skin is dynamic and subject to constant tension from the surrounding muscles of facial expression including the orbicularis oculi, corrugators, and procerus, which likely accelerate rhytid development.

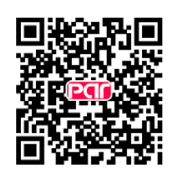


Both the intrinsic factors mentioned above and various extrinsic and environmental factors contribute to accelerated signs of aging in this region. Sun damage is an important contributor, leading to the development of deep rhytids, hyperpigmentation, and a leathery appearance ${ }^{[2]}$. This is exacerbated by the fact that the skin of the eyelids is rarely sufficiently protected even when sunscreen is applied. UV exposure has been shown to lead to the development of reactive oxygen species, which stimulates a milieu that both accelerates collagen breakdown and down-regulates collage synthesis. Smoking is another important modifiable factor that contributes to premature and accelerated skin aging through a similar free-radical mediated mechanism ${ }^{[4,5]}$.

Techniques for rejuvenation of this area include surgery (i.e., blepharoplasty, brow lift), injectables (botulinum toxin, dermal filler), laser resurfacing and photorejuvenation, and topical therapy. In this article we will focus specifically on the techniques and indications for use of toxins and fillers in the periocular region with the aim to provide the reader with a primer for their use based on our own clinical experience.

\section{NON-SURGICAL REJUVENATION OF THE PERIOCULAR AREA: TOXINS}

\section{Overview}

Of the 7 known serotypes of botulinum toxin, A and B are currently available for clinical use. These compounds, produced by the bacterium Clostridium botulinum, effect a flaccid paralysis by preventing presynaptic acetylcholine releas ${ }^{[6]}$. There are 3 commercially available varieties of botulinum toxin A currently available in the United States: Botox ${ }^{\oplus}, \mathrm{Xeomin}^{\circledR}$ and Dysport ${ }^{\oplus}$, which vary in their molecular weight, concentration, and chemical composition [Table 1].

\section{Preparation and administration}

Each preparation comes as a powder requiring reconstitution. Although the FDA recommends preservative free saline, many injectors favor preserved saline as it may be stored longer and the favorable $\mathrm{pH}$ leads to a less painful injection, which has been concluded based both on personal experience as well as scientific studies $^{[7]}$. Both Botox ${ }^{\oplus}$ and Xeomin ${ }^{\circledR}$ are portioned into 50 or 100 unit vials, while Dysport ${ }^{\circledR}$ is packaged in 300 unit increments. Dilution is at the discretion of the practitioner, however we prefer $1 \mathrm{~mL}$ saline per 100 unit vial of Botox ${ }^{\oplus}$ or Xeomin', and $3 \mathrm{~mL}$ per vial of Dysport ${ }^{\circ}$, thus leading to a concentration of 5 units per $0.1 \mathrm{~mL}$ of reconstituted Botox ${ }^{\diamond}$ and $\mathrm{Xeomin}^{\bullet}$ and 12 units per $0.1 \mathrm{~mL}$ of Dysport ${ }^{\oplus}$, standardizing administration techniques.

The dispersion area of each injected aliquot is roughly $1 \mathrm{~cm}^{2}$. We have found that the Becton Dickenson Ultra Fine II diabetic syringe ( $0.3 \mathrm{~mL}$ syringe with $31 \mathrm{~g}$ needle) works particularly well for toxin injection, as there is no hub and thus no wasted product and the needle is very small. Results begin to emerge 1-3 days postinjection, and mature for about 7-14 days afterward. Typically repeat injection is required every 3-4 months. Contraindications to toxin injection include pregnancy, breastfeeding, injection site infection, allergy or hypersensitivity to any component in the formulation, or neuromuscular junction disorders such as myasthenia gravis, amyotrophic lateral sclerosis, or myopathies.

\section{Techniques and tips by location}

"11" lines or frown lines [Figure 1] - glabellar toxin injection targets the medial depressors of the brow (procerus, corrugator supercilii, depressor supercilii, and medial orbicularis) to address rhytids in this region. Treatment of this muscle group also results in a net lift of the medial brow at rest. We aim to inject at the depth of the targeted muscle beneath the subcutaneous layer. There are several potential complications attendant on injection in this area. The first is accidental injection of the frontalis instead of the glabellar muscles, which may lead to brow ptosis rather than elevation (the frontalis being the only medial brow elevator). Eyelid ptosis may also occur if the levator palpebrae superioris is affected. It has been hypothesized that toxin may travel down the anatomical pathway forged by the supratrochlear neurovascular bundle, thus 
Table 1. Properties of commercially available botulinum toxin products

\begin{tabular}{llcl}
\hline & Botox onabotulinumtoxinA & Dysport abobotulinumtoxinA & Xeomin incobotulinumtoxinA \\
\hline Molecular weight & $900 \mathrm{kD}$ & $\geq 300 \mathrm{kD}$ & $150 \mathrm{kD}$ \\
Preparation & Vacuum dried $\mathrm{NaCl}+$ albumin & Lyophilized lactose + albumin & Lyophilized albumin + sucrose \\
$\mathrm{pH}$ & Neutral & Neutral & Neutral \\
Vial size & 50 and 100 units & 300 units & 50 and 100 units \\
Storage temperature & $2-8{ }^{\circ} \mathrm{C}$ & $2-8{ }^{\circ} \mathrm{C}$ & Room Temperature \\
Other & & Contraindicated if cow milk & Retention of particles under lid requires tilting \\
& & protein allergy & after reconstitution to capture all product \\
\hline
\end{tabular}

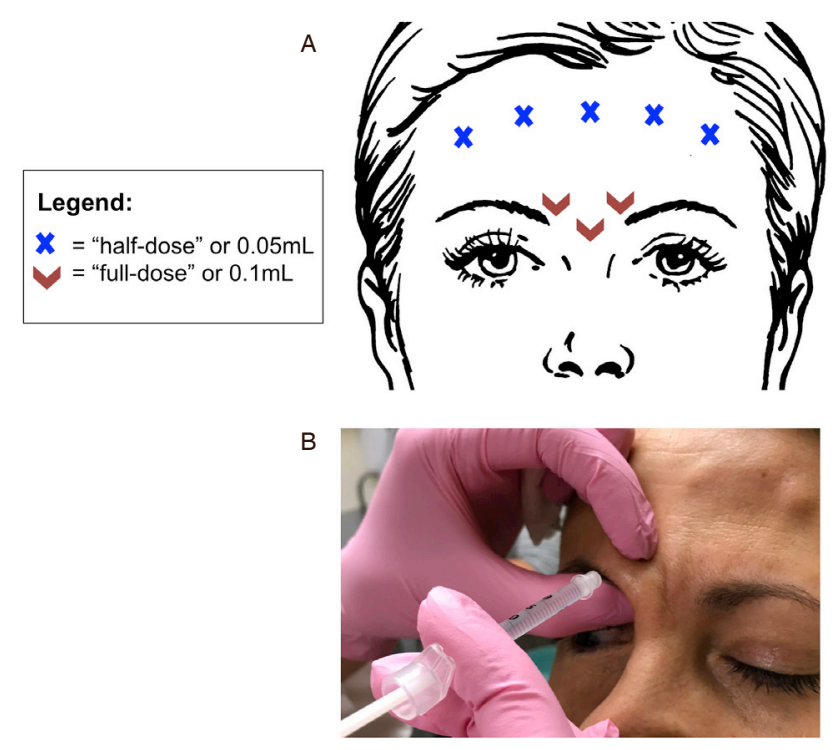

Figure 1. A: Botulinum toxin injection for forehead wrinkles and frown lines. Glabellar injections may be performed without treatment of the frontalis, but frontalis injections must always be accompanied with glabellar treatment to avoid brow ptosis. Volumes referenced are independent of neurotoxin variety and assume dilution as specified in the body of the text (i.e., $1 \mathrm{~mL}$ saline per 100 unit vial of Botox ${ }^{\circledR}$ or $\mathrm{Xeomin}^{\circledR}$ and $3 \mathrm{~mL}$ per vial of Dysport ${ }^{\circledR}$ ); B: demonstration of injection technique whereby pinching and elevating the tissue protects the underlying neurovascular bundle. While these figures offer a guide, dosing may vary between patients according to their musculature and anatomy

we pinch and elevate the skin during the two lateral injections in this area to occlude this potential pathway and protect the orbit [Figure 1B]. If blepharoptosis should occur, apraclonidine $0.5 \%$ eye drops may be used up to three times daily to raise the eyelid approximately $2 \mathrm{~mm}$ via stimulation of the sympathetically innervated Müller's muscle until the effects of the toxin wear off.

Forehead wrinkles [Figure 1] - the frontalis is the major elevator of the brow and is responsible for creating the horizontal rhytids of the forehead. We typically inject roughly $2 / 3$ of the way up the forehead (always staying at or above the midline) to limit the risk of inducing brow ptosis. Injections in this area should always be accompanied by glabellar injections, otherwise the unopposed action of the brow depressors will drop the brow and lead to an unattractive, angry appearing facial expression. It is also important to carry injections far enough laterally so that the lateral edges of the frontalis are also treated, otherwise preserved action in this area will elevate the lateral brow, leading to peaking (colloquially referred to as "Spocking", named for the post-procedure similarity in appearance to the fictional captain). Finally, in patients with significant brow or eyelid ptosis or dermatochalasis who depend on their frontalis for brow elevation, it is wise to avoid injections in this area altogether.

Crow's feet or laugh lines [Figure 2] - injection of the lateral orbicularis oculi muscle may be used to address fine lines lateral and inferior to the eye. It is important to inject on a superficial plane, just below the skin, 


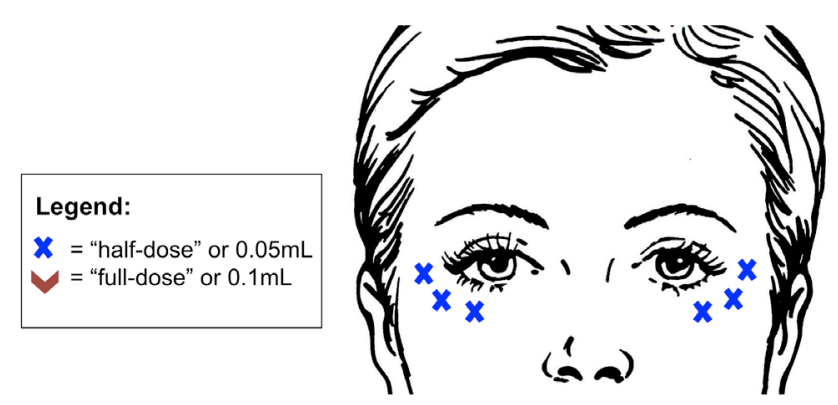

Figure 2. Botulinum toxin injection for crow's feet. It is important not to extend medial to the mid-pupillary line to avoid lower lid ectropion. Lateral injection should be placed approximately $1 \mathrm{~cm}$ lateral to the lateral canthus. Volumes referenced are independent of neurotoxin variety and assume dilution as specified in the body of the text (i.e., $1 \mathrm{~mL}$ saline per 100 unit vial of Botox ${ }^{\circledR}$ or $\mathrm{Xeomin}^{\circledR}$ and $3 \mathrm{~mL}$ per vial of Dysport ${ }^{\circledR}$ ). While these figures offer a guide, dosing may vary between patients according to their musculature and anatomy

to avoid bruising and avoid untargeted muscles. A small welt should be visible after injection similar to a tuberculin skin test. Potential concerns with injections of this area include bruising, as mentioned above, as well as iatrogenic lower lid ectropion if injections are positioned medial to the mid-pupillary line. Injections in this area may also exacerbate symptoms of dry eye in patients with a known history. Finally, if injected too deeply or too inferiorly, muscles of the mid-face may be affected, which may cause an asymmetrical smile.

The shape of the brow may also be subtly modified with toxin injection. Lateral injection of the orbicularis may give 1-2 $\mathrm{mm}$ of lift to the lateral brow [Figure 3]. This may be combined with filler to further shape and fill out this region. The appearance of larger eyes may also be achieved by injection of the pretarsal orbicularis, which widens the palpebral fissure by weakening the pretarsal muscle [Figure 3]. This injection should also target a superficial plane. Again, one must use caution in patients with a history of dry eye, as this may exacerbate their symptoms.

\section{NON-SURGICAL REJUVENATION OF THE PERIOCULAR AREA: FILLERS}

\section{Overview}

Facial aging is also characterized by soft tissue volume loss of the periorbital area. Although neurotoxins in isolation may be sufficient to ameliorate dynamic rhytids in younger patients, it is insufficient to address static changes caused by volume loss in the dermis and underlying soft tissue. Thus, botulinum toxin in combination with dermal fillers is an excellent option for non-invasive rejuvenation in patients with deep, static rhytids. The use of fillers in combination with neurotoxins has the added benefit of approximately doubling the half-life of the filler ${ }^{[8]}$.

Dermal fillers are used to restore facial fullness and volume. Common materials include hyaluronic acid, calcium hydroxylapatite, poly-L-lactic acid, polymethylmethacrylate, and autologous fat ${ }^{[9]}$. For periorbital rejuvenation, we almost exclusively use hyaluronic acid products. Hyaluronic acid is a naturally occurring polysaccharide found in the connective tissue of all living species and so is very well tolerated. It is hydrophilic, and this ability to bind water molecules enables its natural contribution to skin turgor and dermal volume. With aging, natural levels of dermal hyaluronans diminish ${ }^{[8]}$. In terms of cosmetic injectables, of particular value is the ability to dissolve this material with hyaluronidase in the event of the rare but very real potential complication of vascular occlusion. This may also be useful if there is need for revision in the case of suboptimal cosmetic outcome, such as irregular surface contour or Tyndall effect, especially important in the delicate and unforgiving periocular region. Given these factors, hyaluronic acid is our filler of choice in this area. There are a variety of types of hyaluronic acid fillers commercially available [Table 2]. The three major brands in the United States are the Restylane family, Juvederm family 


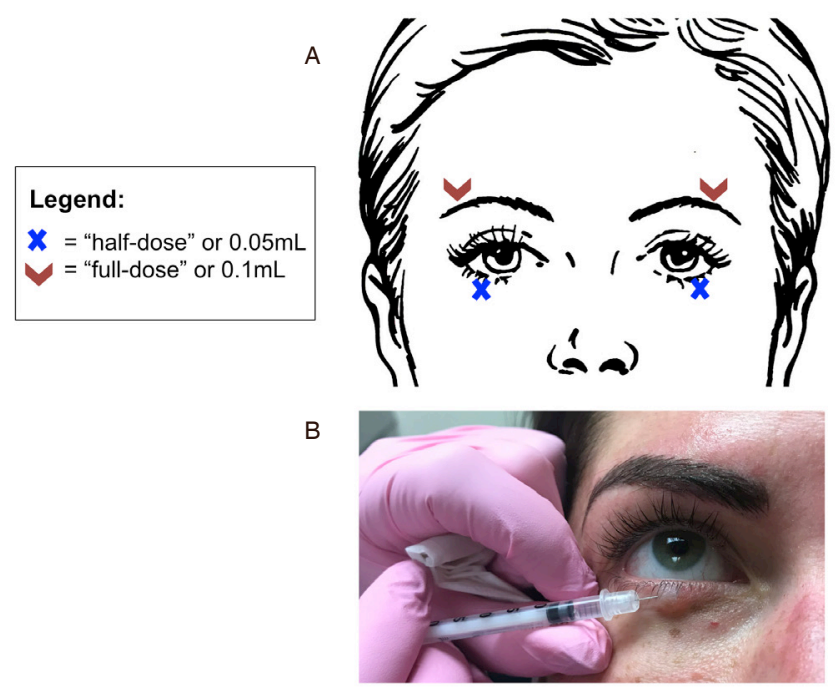

Figure 3. A: Botulinum toxin injection for lateral brow lift and pretarsal orbicularis injection to widen the palpebral fissure. Volumes referenced are independent of neurotoxin variety and assume dilution as specified in the body of the text (i.e., $1 \mathrm{~mL}$ saline per 100 unit vial of Botox ${ }^{\circledR}$ or $\mathrm{Xeomin}^{\circledR}$ and $3 \mathrm{~mL}$ per vial of Dysport); B: proper technique and position for the pretarsal orbicularis injection. While these figures offer a guide, dosing may vary between patients according to their musculature and anatomy

of products, and Belotero ${ }^{\circ}$, each of which offer multiple formulations that vary in concentration, particle size, and cross-linking. In general, products with a higher concentration of hyaluronic acid and higher degree of crosslinking are thought to have a higher viscosity and stiffness $\left(G^{\prime}\right)$ and greater duration of effect ${ }^{[10]}$.

\section{Preparation and administration}

Most dermal fillers come preloaded in a $1 \mathrm{~cm}^{3}$ syringe and include the appropriate needle size. Most are made with powder lidocaine to minimize discomfort, but the addition of topical anesthetic for 15-20 min prior to injection is beneficial for the patient.

\section{Injection techniques}

There are four basic techniques commonly used when injecting dermal filler. Choice of technique depends on the location of injection and the complaint being addressed. The first, threading, is the linear application of a continuous line of filler, typically (although not exclusively) injected in a retrograde fashion. Two additional methods build upon this fundamental technique. The crosshatching technique employs continuous lines applied in an overlapping horizontal and vertical pattern to build volume. Fanning is another technique utilizing multiple continuous lines of filler, this time in a fan shaped projection, where multiple lines emanate from a single point of entry as the needle is advanced repeatedly in a radial fashion without withdrawing from the skin. Finally, the serial puncture method involves the delivery of multiple discreet aliquots of product, each with a separate injection ${ }^{[11]}$. Threading may be the best choice for discrete linear rhytids, such as those in the frontalis or glabellar area. Addressing deeper deformities such as the tear trough may be best approached with a serial puncture technique. Larger areas of treatment such as the temple or forehead may require fanning or cross-hatching to build volume, depending on the patient.

\section{Techniques and tips by location}

Tear trough [Figure 4] - this prominence of the lid/cheek junction (also known as the nasojugal or palpebralmalar groove), has historically been very difficult to address with satisfactory results. The development of this deformity is commonly thought to involve both volume loss at the level of the cheek/lid junction as well as increasing prominence of the overlying fat $\mathrm{pad}^{[12]}$. The goal of filler injections in this region is to smooth this transition. This area is the most unforgiving area to inject, so care and conservative measures must be 
Table 2. Summary of hyaluronic acid dermal fillers currently commercially available in the United States. All except for Belotero Balance contain lidocaine

\begin{tabular}{llcc}
\hline Product $^{\circ}$ & \multicolumn{1}{c}{ Site } & HA concentration $\mathbf{( m g} / \mathbf{m L}$ ) & Duration (approximate months) \\
\hline Belotero Balance & Superficial - mid-dermis & 22.5 & 6 \\
Restylane-L & Superficial - mid-dermis & 20 & 6 \\
Restylane Silk & Superficial - sub-mucosal & 20 & 6 \\
Restylane Lyft & Medium - deep & 20 & 9 \\
Restylane Refyne & Medium - deep & 20 & 12 \\
Restylane Defyne & Medium - deep & 20 & 12 \\
Juvederm Ultra XC & Superficial - medium & 24 & 12 \\
Juvederm Volbella & Superficial - medium & 15 & $12-18$ \\
Juvederm Vollure & Medium - deep & 17.5 & $12-18$ \\
Juvederm Voluma XC & Medium - deep & 20 & $12-18$ \\
Juvederm Ultra Plus XC & Medium - deep & 24 & 18 \\
\hline
\end{tabular}

HA: hyaluronic acid

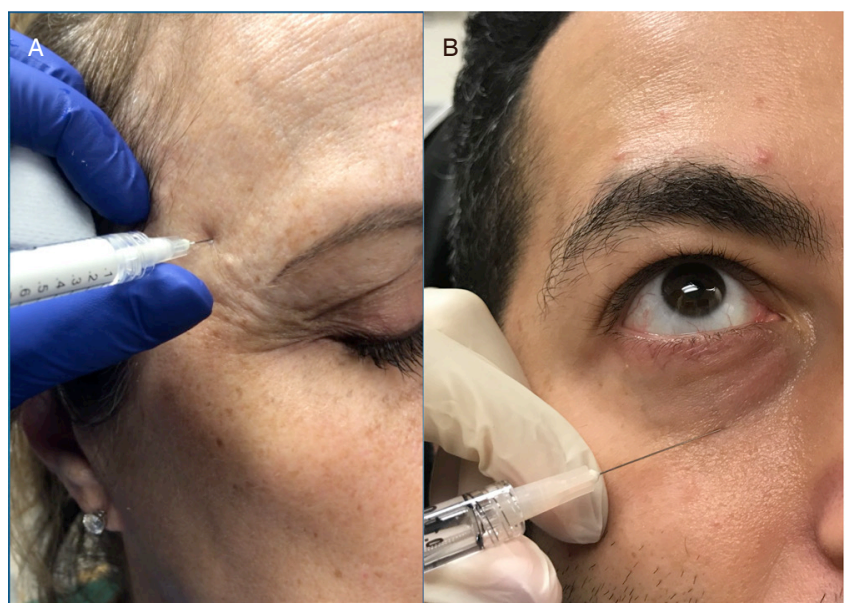

Figure 4. Volume restoration with dermal fillers. Common periocular treatment areas include the temple (A) and tear trough (B)

taken. The authors' choice of filler for this area is a hyaluronic acid with low water affinity, as chronic fluid collection can be a problem leading to the need for surgical excision. We most commonly use Restylane, but have also used Restylane Refyne $e^{\circ}$, Belotero ${ }^{\circ}$, Juvederm Volbella ${ }^{\circ}$, and Juvederm Vollure ${ }^{\circledast}$ in this area. In terms of technique, we introduce the needle bevel down and advance to the periosteum of the inferior orbital rim in order to achieve a sub-orbicularis plane. Deep injection avoids visible product and irregular contour. Product is delivered just above the periosteum in multiple small boluses working from medial to lateral. Prior to injection, it is important to palpate the orbital rim to avoid advancing past this barrier and into the orbit. If midface filler is to be applied, this should be performed prior to tear trough injections, as midface volume often decreases the appearance of the tear trough. It is better to under correct than overcorrect in this delicate area; typically injections are 0.3-0.4 $\mathrm{mL}$ per session, per side ${ }^{[8]}$.

Superior sulcus - hollowing of the superior sulcus is one of the earliest signs of aging in the periorbital region ${ }^{[13]}$. Injection should target the underside of the superior orbital rim, again advancing to bone and aiming for a sub-orbicularis plane. Subcutaneous injection is also possible, although more superficial injections are at a higher risk of poor cosmetic outcome. The supraorbital notch should be palpated prior to injection to avoid compromising the eponymous nerve; likewise it is important to avoid injecting into the eyelid, staying above the superior sulcus at all times. Take care not to advance the needle far into the orbit, as the risk of damage to important structures and retrobulbar hematoma will increase. 
Temple - volume loss in the temples leads to prominence of the bony orbital rim and lateral brow ptosis ${ }^{[14]}$. A more robust product with a high G', such as Juvederm Voluma ${ }^{\oplus}$, Restylane Lyft ${ }^{\circ}$, or Radiesse (a calcium hydroxylapatite product), is preferable in this area. When introducing filler in this region, care must be taken to avoid both the blood vessels and branches of the facial nerve coursing through this area. Recall that the path of the facial nerve as it courses through the superficial temporal fascia may be roughly estimated by drawing a line from $0.5 \mathrm{~cm}$ inferior to the tragus to $1.5 \mathrm{~cm}$ superior to the lateral brow ${ }^{[15]}$. Injection should be initiated in the area of greatest volume loss and typically at least $1 \mathrm{~cm}$ lateral to the lateral orbital rim. A deep plane under the fascia of the temporal muscle on the periosteum is generally safe and also provides a nice cosmetic result. Injection volume varies from less than 1 to over $2 \mathrm{~mL}$ per side depending on the degree of correction required.

Filler may also be used to address static rhytids in combination with toxin injection, as well as to restore volume and arch to the lateral brow, crows' feet and glabella.

\section{Potential complications}

Complications of dermal filler injections include Tyndall effect (a bluish hue to the skin resulting from superficial filler injection), irregular surface contour, hypersensitivity reaction and/or granulomatous inflammation, and the development of festoons or chronic fluid collections. The most serious potential side effects include vascular occlusion leading to tissue necrosis or worse, blindness from retrobulbar hemorrhage or central retinal artery occlusion. In general, proper injection technique and depth minimize the risk of poor cosmetic outcomes such as the Tyndall effect or contour irregularities. As mentioned above, the choice of filler with low water affinity is important to avoiding the development of chronic fluid collections. Vascular occlusions are discussed in more depth below.

\section{Vascular occlusion and ocular ischemia: the most devastating potential complications of dermal filler injection}

Intra-arterial injection of filler may result in vascular occlusion with either anterograde or retrograde embolus of product. Local embolus can lead to soft tissue necrosis or a distant embolus can lead to permanent blindness. If the force of intra-arterial injection is greater than mean arterial blood pressure, retrograde flow can potentially introduce filler into the ophthalmic or central retinal artery, or long and short posterior ciliary arteries, leading to visual loss. More proximal flow may introduce filler into the middle cerebral artery resulting in cerebral infarction. If tissue necrosis should occur, there are several important steps to take to restore perfusion. First, warm compresses can be used to promote local vasodilation (this low risk treatment is theoretically helpful, although there is no data to support its efficacy and there is some worry that vasodilation can encourage an embolus to flow further distally). Topical nitroglycerin may also be used to promote vasodilation, with the same theoretical advantages and disadvantages. Again, there is no evidence proving its effectiveness ${ }^{[16]}$. Second, the area should be flooded with injected hyaluronidase; this is the only proven treatment for this complication. Generally, subcutaneous injection is thought to be sufficient, but intra-arterial administration may also be considered if possible and time allows. Practically, reintroduction of the needle into the occluded artery may not be possible, and prior studies have shown that extravascular hyaluronidase is effective on the intravascular material ${ }^{[17]}$.

It is important to have a large volume of hyaluronidase easily accessible for prompt treatment; we recommend at least 10 (unexpired) vials. There are a variety of hyaluronidase formulations available, which may be of human recombinant or animal derived origin. Characteristics such as storage parameters, preservatives, units per vial, and reconstitution requirements vary. Although different types of filler may respond to hyaluronidase differently, there is no evidence to suggest that a specific hyaluronidase formulation is more effective ${ }^{[18]}$. Although there are rare reports of allergic reactions to hyaluronidase, skin 
testing is not performed in this acute setting, but may be considered if administered for a non-emergent cause (i.e., cosmetic revision of prior injection) ${ }^{[19]}$. Multiple round of injections of hyaluronidase flooding the ischemic site may be necessary, and the patient should remain in-office until reperfusion is achieved. In the event of occlusion of the ophthalmic circulation, treatment is more complicated, and no treatment has been proven effective. Potential options include a retrobulbar hyaluronidase injection, which comes with a fair amount of risk and must be administered by a trained provider, intra-arterial hyaluronidase injection, and attempts to increase the perfusion pressure of the retina and optic nerve by lowering intraocular pressure ${ }^{[16]}$. Prompt referral to an ophthalmologist should be made. For a more complete discussion of the management of various types of filler complications including vascular occlusion, the authors refer you to the review article by Hwang ${ }^{[16]}$. Given the gravity of these complications, prevention is paramount and conservative injection techniques with background knowledge are essential.

Tips for prevention:

1. Needle positioning: the authors recommend the needle be introduced in a direction perpendicular to arterial vessels OR parallel to vessels with needle pointed in the direction of distal flow;

2. Use a small needle, but not too small so as to avoid arteriolar cannulation;

3. Withdraw slightly after introducing the needle;

4. Inject slowly with low flow, low pressure technique so as to avoid introducing filler at a pressure greater than mean arterial pressure;

5. Severe pain is NOT normal; stop if this occurs;

6. Prior to glabellar and superior sulcus injections, digital pressure is applied to the supratrochlear and supraorbital neurovascular bundles, which theoretically occludes the vessels, raises intra-arterial pressure, and reduces the risk of retrograde flow should intra-arterial cannulation occur.

\section{CONCLUSION}

The anatomy and dynamics of the periorbital region make this area particularly vulnerable to aging and particularly complex to treat. Botulinum toxins and fillers, especially when used in combination, offer an excellent approach to minimally invasive rejuvenation of this area. It is important to consider their use within the context of a comprehensive approach to rejuvenation of this area. Additional techniques including laser resurfacing and photorejuvention may be used as indicated for each individual patient and a combination approach is often required for optimal results. As with any procedure, a thorough understanding of the underlying anatomy and sound injection technique are important to achieve optimal results and avoid complications.

\section{DECLARATIONS}

\section{Authors' contributions}

Conceptualization, writing, and editing of this manuscript: Lee WW, Levitt AE

\section{Availability of data and materials}

None.

\section{Financial support and sponsorship}

None.

\section{Conflicts of interest}

Lee WW acts as a consultant for Allergan, Merz, Galderma, Ophthalmology Web, and Mallinckrodt. Levitt $\mathrm{AE}$ has no conflicts of interest to declare. 


\section{Ethical approval and consent to participate}

Not applicable.

\section{Consent for publication}

All photographs were obtained and used with consent.

\section{Copyright}

(c) The Author(s) 2018.

\section{REFERENCES}

1. Coleman SR, Grover R. The anatomy of the aging face: volume loss and changes in 3-dimensional topography. Aesthet Surg J 2006;26:S4-9.

2. Helfrich YR, Sachs DL, Voorhees JJ. Overview of skin aging and photoaging. Dermatol Nurs 2008;20:177-83; quiz 84.

3. Ha RY, Nojima K, Adams WP Jr, Brown SA. Analysis of facial skin thickness: defining the relative thickness index. Plast Reconstr Surg 2005;115:1769-73.

4. Keaney TC. Aging in the male face: intrinsic and extrinsic factors. Dermatol Surg 2016;42:797-803.

5. Morita A. Tobacco smoke causes premature skin aging. J Dermatol Sci 2007;48:169-75.

6. Dressler D, Saberi FA, Barbosa ER. Botulinum toxin: mechanisms of action. Arq Neuropsiquiatr 2005;63:180-5.

7. Alam M, Dover JS, Arndt KA. Pain associated with injection of botulinum A exotoxin reconstituted using isotonic sodium chloride with and without preservative: a double-blind, randomized controlled trial. Arch Dermatol 2002;138:510-4.

8. Coleman KR, Carruthers J. Combination therapy with BOTOX and fillers: the new rejuvnation paradigm. Dermatol Ther 2006;19:177-88.

9. Raspaldo H, Gassia V, Niforos FR, Michaud T. Global, 3-dimensional approach to natural rejuvenation: part 1 - recommendations for volume restoration and the periocular area. J Cosmet Dermatol 2012;11:279-89.

10. Bentkover SH. The biology of facial fillers. Facial Plast Surg 2009;25:73-85.

11. Brandt FS, Cazzaniga A, Strangman N, Coleman J, Axford-Gatley R. Long-term effectiveness and safety of small gel particle hyaluronic acid for hand rejuvenation. Dermatol Surg 2012;38:1128-35.

12. Lambros VS. Hyaluronic acid injections for correction of the tear trough deformity. Plast Reconstr Surg 2007;120:74S-80S.

13. Liew S, Nguyen DQ. Nonsurgical volumetric upper periorbital rejuvenation: a plastic surgeon's perspective. Aesthetic Plast Surg 2011;35:319-25

14. Ross JJ, Malhotra R. Orbitofacial rejuvenation of temple hollowing with Perlane injectable filler. Aesthet Surg J 2010;30:428-33.

15. Pitanguy I, Ramos AS. The frontal branch of the facial nerve: the importance of its variations in face lifting. Plast Reconstr Surg 1966;38:352-6.

16. Hwang CJ. Periorbital Injectables: understanding and avoiding complications. J Cutan Aesthet Surg 2016;9:73-9.

17. DeLorenzi C. Transarterial degradation of hyaluronic acid filler by hyaluronidase. Dermatol Surg 2014;40:832-41.

18. Rao V, Chi S, Woodward J. Reversing facial fillers: interactions between hyaluronidase and commercially available hyaluronic-acid based fillers. J Drugs Dermatol 2014;13:1053-6.

19. Landau M. Hyaluronidase caveats in treating filler complications. Dermatol Surg 2015;41 Suppl 1:S347-53. 